Methods: A temporary recommendation for use for infliximab in refractory TA was approved by the French National Drug Authorities (April 2014). Infliximab was administered to patients in case of disease activity with a NIH score $\geq 2$ despite conventional therapy. Data regarding patient's clinical, laboratory, imaging and treatments were obtained at baseline, and at each following visit until last visit (October 2017). TA activity was evaluated according to $\mathrm{NIH}$ criteria and GC requirement throughout the study.

Results: Twenty-three patients were enrolled, including 19 female. The median age at inclusion was 33 years (Interquartile range, IQR: 23-44 years). At baseline, $17(74 \%)$ patients were treated with GCs, at a median dose of $10 \mathrm{mg} / \mathrm{day}$ (IQR: 0-21) of prednisone-equivalent. After a median follow-up of 36.9 months (IQR: 10-58.7), improvement of $\geq 1 \mathrm{NIH}$ criterion of TA activity was achieved for 14/22 (64\%) patients. The median GC dose was $8 \mathrm{mg} /$ day (IQR: 7-10) at 6 months; $5 \mathrm{mg} /$ day (IQR: 0-8) at 12 months and $0 \mathrm{mg} /$ day (IQR: 0-5) at 36 months of follow-up. Overall, infliximab originator had a significant GC-sparing effect between baseline and last follow-up $(p=0.009)$.

Conclusion: This multicenter open-label cohort study suggests that infliximab originator is an effective GC-sparing treatment for TA refractory to conventional therapy.

Disclosure of Interests: None declared

DOI: 10.1136/annrheumdis-2020-eular.1241

\begin{tabular}{|l}
\hline AB0511 \\
INTERNATIONAL CONSENSUS ON ANCA TESTING \\
AND INTERPRETATION BEYOND SYSTEMIC \\
VASCULITIS
\end{tabular}

S. Moiseev $^{1}$, J. W. Cohen Tervaert ${ }^{2,3}$, Y. Arimura ${ }^{4}$, D. Bogdanos ${ }^{5}$, C. Elena ${ }^{6}$, J. Damoiseaux ${ }^{3}$, M. Ferrante ${ }^{7}$, L. F. Flores-Suárez ${ }^{8}$, M. Fritzler ${ }^{9}$, P. Invernizzi ${ }^{10}$, D. Jayne ${ }^{11}$, J. C. Jennette ${ }^{12}$, M. Little ${ }^{13}$, S. P. Mcadoo ${ }^{14}$, P. Novikov ${ }^{1}$, C. D. Pusey ${ }^{14}$, A. Radice ${ }^{15}$, A. D. Salama ${ }^{16}, J$. Savige ${ }^{17}$, M. Segelmark ${ }^{18,19}$, Y. Shoenfeld ${ }^{1,20,21}$, R. A. Sinico ${ }^{10}$, M. J. R. De Sousa ${ }^{22}$, U. Specks ${ }^{23}$, B. Terrier ${ }^{24}$, A. Tzioufas ${ }^{25}$, S. Vermeire ${ }^{7}$, M. H. Zhao ${ }^{26}$, X. Bossuyt ${ }^{7} .{ }^{1}$ Sechenov First Moscow State Medical University, Moscow, Russian Federation; ${ }^{2}$ University of Alberta, Edmonton, Canada; ${ }^{3}$ Maastricht University Medical Center, Maastricht, Netherlands; ${ }^{4}$ Kyorin University School of Medicine, Tokyo, Japan; ${ }^{5}$ University of Thessaly, Larissa, Greece; ${ }^{6}$ University of Tübingen, Kirchheim-Teck, Germany; ${ }^{7}$ University Hospitals Leuven, Leuven, Belgium; ${ }^{8}$ Instituto Nacional de Enfermedades Respiratorias, Mexico City, Mexico; ${ }^{9}$ University of Calgary, Calgary, Canada; ${ }^{10}$ Università degli Studi di Milano-Bicocca, Monza, Italy; ${ }^{11}$ University of Cambridge, Cambridge, United Kingdom; ${ }^{12}$ University of North Carolina, Chapel Hill, United States of America; ${ }^{13}$ Trinity Health Kidney Centre, Dublin, Ireland; ${ }^{14}$ Imperial College London, London, United Kingdom; ${ }^{15}$ San Carlo Borromeo Hospital, Milan, Italy; ${ }^{16}$ Royal Free Hospital, London, United Kingdom; ${ }^{17}$ University of Melbourne, Melbourne, Australia; ${ }^{18}$ Lund University, Lund, Sweden; ${ }^{19}$ Skane University Hospital, Lund, Sweden; ${ }^{20}$ Sheba Medical Center, Tel Hashomer, Israel; ${ }^{21}$ Tel Aviv University, Tel Aviv, Israel; ${ }^{22}$ Centro de Medicina Laboratorial Germano de Sousa, Lisbon, Portugal; ${ }^{23}$ Mayo Clinic, Rochester, United States of America; ${ }^{24} \mathrm{Hop}$ ital Cochin, Paris, France; ${ }^{25}$ National and Kapodistrian University of Athens, Athens, Greece; ${ }^{26}$ Peking University First Hospital, Beijing, China

Background: ANCA can be detected in sera from patients with autoimmune, inflammatory, infectious or neoplastic diseases.

Objectives: To issue a Consensus Statement on ANCA testing and interpretation beyond systemic vasculitis.

Methods: This Statement was prepared by a group of experts, based on the results of a comprehensive search in PubMed.

Results: In certain settings beyond systemic vasculitis, ANCA may have diagnostic, clinical, and/or prognostic relevance. Testing for PR3- and MPOANCA by specific immunoassays should be performed in any patient with clinical features suggesting ANCA-associated vasculitis and in patients with anti-GBM disease and idiopathic interstitial pneumonia. Routine ANCA testing is not recommended in patients with connective tissue diseases (CTD), autoimmune liver diseases, inflammatory bowel diseases, infections, and/ or malignancy unless there is evidence for small vessel vasculitis. ANCA testing by specific immunoassays may be useful in patients with rheumatoid arthritis, systemic sclerosis or primary Sjögren's syndrome who have kidney disease with a nephritic sediment or in patients with systemic lupus erythematosus if a kidney biopsy shows prominent necrotizing and crescentic lesions or proliferative lupus nephritis. ANCA testing may be justified in patients with suspected autoimmune hepatitis type 1, who do not have conventional disease-related autoantibodies, or in patients with inflammatory bowel diseases in case of diagnostic uncertainty to discriminate ulcerative colitis from Crohn's disease. In these cases, ANCA should be tested by indirect immunofluorescence since target antigens are not well characterized. ANCA against bactericidal/permeability-increasing protein may be a biomarker for deteriorating lung function and a poor prognosis in patients with cystic fibrosis.

Conclusion: ANCA testing is clinically relevant not only in patients with manifestations suggesting systemic vasculitis, but also in patients with certain othe disorders, particularly in patients with anti-GBM disease or idiopathic interstitial pneumonia

Disclosure of Interests: Sergey Moiseev Grant/research support from: This work was supported by the 5-100 Project, Sechenov University, Moscow, Jan Willem Cohen Tervaert: None declared, Yoshihiro Arimura: None declared, Dimitrios Bogdanos: None declared, Csernok Elena: None declared, Jan Damoiseaux: None declared, Marc Ferrante: None declared, Luis Felipe Flores-Suárez: None declared, Marvin Fritzler: None declared, Pietro Inv ernizzi: None declared, David Jayne Grant/research support from: ChemoCentryx, GSK, Roche/Genentech, Sanofi-Genzyme, Consultant of: Astra-Zeneca, ChemoCentryx, GSK, InflaRx, Takeda, Insmed, Chugai, Boehringer-Ingelheim, J. Charles Jennette: None declared, Mark Little: None declared, Stephen P. McAdoo: None declared, Pavel Novikov Grant/research support from: This work was supported by the 5-100 Project, Sechenov University, Moscow, Charles D. Pusey: None declared, Antonella Radice: None declared, Alan D. Salama: None declared, Judith Savige: None declared, Mårten Segelmark: None declared, Yehuda Shoenfeld: None declared, Renato Alberto Sinico: None declared, Maria Jose Rego de Sousa: None declared, Ulrich Specks: None declared, Benjamin Terrier: None declared, Athanasios Tzioufas: None declared, Severine Vermeire: None declared, Ming-hui Zhao: None declared, Xavier Bossuyt: None declared

DOI: 10.1136/annrheumdis-2020-eular.219

\section{AB0512 ALLERGIC PROFILE AND ALLERGEN- SPECIFIC IMMUNOTHERAPY IN EOSINOPHILIC GRANULOMATOSIS WITH POLYANGIITIS (EGPA): A SINGLE CENTER OBSERVATIONAL STUDY}

L. Moroni ${ }^{1,2}$, A. Cariddi ${ }^{1}$, S. Sartorelli ${ }^{1}$, E. Della Torre ${ }^{1,2}$, T. Germanò $^{2}$, G. A. Ramirez ${ }^{1,2}$, E. Bozzolo', M. R. Yacoub ${ }^{1}$, L. Dagna ${ }^{1,2} .{ }^{1}$ IRCCS San Raffaele Hospital, Unit of Immunology, Rheumatology, Allergy and Rare Diseases, Milano, Italy; ${ }^{2}$ Vita-Salute San Raffaele University, Milano, Italy

Background: Eosinophilic granulomatosis with polyangiitis (EGPA), previously known as Churg-Strauss syndrome, is a systemic disease characterized by late onset asthma associated with small- and/or medium-size vessel vasculitis besides eosinophil-mediated cytotoxic organ damage. About $20-30 \%$ of patients with EGPA displays allergic manifestations related with inhalant sensitization while prevalence of food and drug allergy is unknown in this context. Moreover, some authors in the past hypothesized in favor of a possible role of allergen-specific immunotherapy (ASIT) as a trigger of disease.

Objectives: Aim of the present study is to establish the prevalence of each category allergen sensitization and to determine whether atopy or specific immunotherapy could influence clinical expression of the disease.

Methods: Our study consisted in a retrospective demographic and clinical data collection regarding EGPA history (including age at diagnosis, organ and tissue involvement, autoantibody profile) and the presence of allergic comorbidities or previous drug hypersensitivity reactions. Patients without either proven allergic reactions or positive tests have been excluded.

Results: Fifty-three (53) patients with definitive diagnosis of EGPA have been included in the analysis among which $25(47.2 \%)$ with chronic respiratory allergy or previous acute allergic reaction. Among allergic patients $15(60 \%)$ resulted sensitized towards inhalants and among them $13(86.7 \%)$ displayed multiple sensitization. Drug allergy affected 13 patients (52\%), food 4 (16\%). Among 15 patients with respiratory allergy, 13 were eligible to allergen-specific immunotherapy (ASIT). Seven (7) subjects underwent ASIT prior EGPA diagnosis with an average time-to-EGPA of 16.2 years. No statistically significant difference was found in terms of sex, age at diagnosis, positivity for or specificity of anti-neutrophil cytoplasm antibodies (ANCA), eosinophil count at onset, pattern of clinical manifestations comparing allergic vs. non-allergic, ASIT vs. non-ASIT, ASIT vs. allergic, ASIT vs. eligible.

Conclusion: Among patients with EGPA allergies are highly prevalent, particularly towards inhalants and drugs. In the great majority of patients multiple sensitization profile is found. Atopy doesn't seem to be associated with specific patterns of disease presentation. The absence of correlation between inhalant ASIT exposure and variation in mode and time of EGPA onset doesn't support the hypothesis of a its potential role in triggering the disease. 


\section{References:}

[1] Berti A et al. Severe/uncontrolled asthma and overall survival in atopic patients with eosinophilic granulomatosis with polyangiitis. Respiratory Medicine 2018; DOI: 10.1016/j.rmed.2018.07.017

[2] Cottin V et al. Respiratory manifestations of eosinophilic granulomatosis with polyangiitis (Churg-Strauss). European Respiratory Journal 2016; DOI: 10.1183/13993003.00097-2016

Disclosure of Interests: Luca Moroni: None declared, adriana cariddi: None declared, Silvia Sartorelli: None declared, Emanuel Della Torre: None declared Tommaso Germanò: None declared, Giuseppe Alvise Ramirez: None declared, Enrica Bozzolo: None declared, Mona-Rita Yacoub: None declared, Lorenzo Dagna Grant/research support from: The Unit of Immunology, Rheumatology, Allergy and Rare Diseases (UnIRAR) received unresctricted research/educational grants from Abbvie, Bristol-Myers Squibb, Celgene, Janssen, Merk Sharp \& Dohme, Mundipharma Pharmaceuticals, Novartis, Pfizer, Roche, Sanofi-Genzyme, and SOBI., Consultant of: Prof Lorenzo Dagna received consultation honoraria from Abbvie, Amgen, Biogen, Bristol-Myers Squibb, Celltrion, Novartis, Pfizer, Roche, Sanofi-Genzyme, and SOBI.

DOI: 10.1136/annrheumdis-2020-eular.1766

\section{AB0513 GLOMERULONEPHRITIS IN LEVAMISOLE- ADULTERATED COCAINE VASCULOPATHY (LACIV): A 51-CASE SERIES}

C. Muñoz ${ }^{1,2,3}$, D. Jaramillo Arroyave ${ }^{1,2,3,4}$, L. Hernandez ${ }^{2}$, L. A. González² G. Vásquez ${ }^{2}$, M. Restrepo Escobar ${ }^{2}$, T. Urrego Callejas ${ }^{2}$, A. Vanegas ${ }^{1,2}$. ${ }^{1}$ Hospital Universitario San Vicente Fundación, Medellín, Colombia;

${ }^{2}$ Universidad de Antioquia, Medellín, Colombia; ${ }^{3}$ IPS Universitaria, Servicios de Salud Universidad de Antioquia, Medellín, Colombia; ${ }^{4}$ Grupo de Epidemiología y Bioestadística Universidad CES, Medellín, Colombia

Background: Up to $88 \%$ of cocaine is tainted with levamisole, anthelmintic withdrawn from the market due to toxicity. Since 2010 LACIV patients, characterized by retiform purpura, ear necrosis, multisystemic compromise and positivity for multiple autoantibodies, have been reported. Renal involvement is the most serious and heterogeneous clinical manifestation.

Objectives: To describe the renal involvement of patients with LACIV.

Methods: We describe the renal manifestations of a 51 case series with LACIV admitted in four high complexity institutions in Colombia from December 2010 to December 2019

Results: All patients were mestizos, with median age of 32.5 years (SD 7.8), the male:female ratio was $4.7: 1$, and the time from symptoms to diagnosis 12 months (IR 32). Nephritis was found in $60.8 \%$, with creatinine elevation in $61 \%$, median $2.0 \mathrm{mg} / \mathrm{dl}$ (IR 3), $87 \%$ had proteinuria, median $3184 \mathrm{mg} /$ day (IR 5735 ), $43 \%$ in nephrotic-range; $93 \%$ had hematuria and $48 \%$ pyuria and cilindruria. Biopsy was performed in 21 patients (64\%), with immune complex mediated extracapillary glomerulonephritis (35\%), immune complex mediated membranoproliferative glomerulonephritis (20\%) pauci-immune proliferative glomerulonephritis $(20 \%)$ membranous glomerulonephritis (15\%), focal and segmental glomerulosclerosis (5\%) and C3 mediated extracapillary glomerulonephritis (5\%). Six patients (19\%) developed end-stage kidney disease. Patients with nephritis had more ear necrosis, retiform purpura, leukopenia, lymphopenia, anemia, hypocomplementemia, anti-PR3 and anti-MPO antibodies, compared to patients without nephritis.

Conclusion: Due to the higher abuse of cocaine and its contamination with levamisole, LACIV is an increasingly reported disease. Although skin manifestations are the most characteristic and prevalent, renal involvement is frequent, clinically and histologically heterogeneous, and potentially serious. Cytopenias, hypocomplementemia and antineutrophil cytoplasmic antibodies could identify patients at risk of nephritis

References:

[1] Collister D, Sathianathan C, Ryz K, Karpinski M, Bernstein K, Gibson IW. ANCA Associated Vasculitis Secondary to Levamisole-Adultered Cocaine with Associated Membranous Nephropathy: A Case Series. Am J Nephrol. 2017;45(3):209-16.

[2] Carlson $A Q$, Tuot DS, Jen K-Y, Butcher B, Graf J, Sam R, et al. Pauci-immune glomerulonephritis in individuals with disease associated with levamisole-adulterated cocaine: a series of 4 cases. Medicine (Baltimore). 2014 Oct;93(17):290-7.

Disclosure of Interests: None declared

DOI: 10.1136/annrheumdis-2020-eular.6596

\section{$\mathrm{AB} 0514$ \\ CALF PAIN, KEY POINT IN THE DIAGNOSIS OF POLYARTERITIS NODOSA}

J. S. Peinado Acevedo ${ }^{1}$, M. Calle ${ }^{2,3}$, A. Medina ${ }^{1}$, D. Jaramillo Arroyave ${ }^{1,4,5,6}$, A. Vanegas ${ }^{1,5}$, L. A. González ${ }^{1}$, G. Vásquez ${ }^{1}$, M. Restrepo Escobar ${ }^{1}$,
C. Muñoz ${ }^{1,4,5} .{ }^{1}$ Universidad de Antioquia, Medellín, Colombia; ${ }^{2}$ Facultad de Medicina Universidad CES, Medell ín, Colombia; ${ }^{3}$ Hospital General, Medellín, Colombia; ${ }^{4}$ IPS Universitaria, Servicios de Salud Universidad de Antioquia, Medellín, Colombia; ${ }^{5}$ Hospital Universitario San Vicente Fundación, Medellín, Colombia; ${ }^{6}$ Grupo de Epidemiología y Bioestadística Universidad CES, Medellín, Colombia

Background: polyarteritis nodosa (PAN) is a primary systemic vasculitis that is becoming a rare disease in part by the decrease in hepatitis $B$ virus (HBV) infection due to widespread vaccination. It is characterized by a full vast constellation of nonspecific clinical manifestations, which sometimes delays and makes it difficult to diagnose. Still, muscle involvement is a feature that could guide the clinician.

Objectives: to describe the main clinical and laboratory characteristics of patients with PAN and to confirm the frequency of muscle involvement.

Methods: retrospective cross-sectional descriptive study of 23 adult patients diagnosed with PAN between January 2011 and December 2018 in two high complexity hospitals in Medellin-Colombia.

Results: twenty-three patients met ACR 1990 classification criteria for PAN, 52\% were men with a median age of 51 (IR 36-60), 78.3\% were newly diagnosed, and only two patients (8.7\%) had HBV infection. General symptoms (found in $95 \%$ of the patients), cutaneous ( $82 \%$ ), and articular (56\%) were the most frequent manifestations. Among systemic symptoms, myalgia, especially calf pain, was the most common characteristic $(78.3 \%)$, followed by weight loss $(73.9 \%)$, fatigue $(69.3 \%)$, and fever (59.3\%). Laboratory findings and severity scores are shown in the table. Angiography was performed in $27.3 \%$ of patients, finding splanchnic (renal, hepatic and splenic) microaneurysms (17.4\%), stenosis (13\%), and renal infarction (4.3\%). Fourteen patients $(61 \%)$ had at least one positive biopsy documenting medium-sized artery vasculitis, mainly skin, muscle, nerve, or both; $9(39 \%)$ had normal or inconclusive biopsy findings. All patients received high daily doses of prednisolone $(50 \pm 16 \mathrm{mg}) ; 52.2 \%$ required cyclophosphamide $30.4 \%$ azathioprine, $17.4 \%$ methotrexate, $8.7 \%$ rituximab, $4.3 \%$ dapsone and 4.3\% plasmapheresis; acetylsalicylic acid was given to half of the patients and only one required antiviral therapy for HBV. With treatment, $87 \%$ improved; $22.7 \%$ had an infection, and $8.7 \%$ of patients died.

Conclusion: myalgia was the main characteristics of our PAN patients, especially in calves, and its presence in patients with other general, skin or articular symptoms should raise the suspect of this vasculitis.

References:

[1] Karadag O, Jayne DJ. Polyarteritis nodosa revisited: a review of historical approaches, subphenotypes and a research agenda. Clin Exp Rheumatol. 2018;36 Suppl 111(2):135-142.

[2] Pagnoux C, Seror R, Henegar C, et al. Clinical features and outcomes in 348 patients with polyarteritis nodosa: a systematic retrospective study of patients diagnosed between 1963 and 2005 and entered into the French Vasculitis Study Group Database. Arthritis Rheum. 2010;62(2):616-626.

Table

\section{Characteristic} PAN patients $(n=23)$

CRP (mean and SD in $\mathrm{mg} / \mathrm{dl}$ )

ESR (mean and SD in $\mathrm{mm} / \mathrm{h}$ )

CPK (median and IR in U/L) normal value $<180$

FFS (mean)

BVAS (median and IR)

$6.3 \pm 8.51$

$84 \pm 38$

$76(66)$

1

PAN: polyarteritis nodosa; CRP: C-reactive protein; ESR: erythrocyte sedimentation rate; $\mathrm{CPK}$ : creatine phosphokinase; FFS: five factor score; BVAS: Birmingham Vasculitis Activity Score; SD: standard desviation; IR (interquartile range)

Disclosure of Interests: None declared DOI: 10.1136/annrheumdis-2020-eular.6578

\begin{tabular}{|l|l}
\hline AB0515 & COMPARISON OF THE PERFORMANCE OF THE 1990 \\
ACR CLASSIFICATION CRITERIA AND THE GIACTA \\
INCLUSION CRITERIA FOR THE CLASSIFICATION OF \\
GCA: RESULTS FROM A SINGLE-CENTRE STUDY.
\end{tabular}

F. Muratore $^{1}$, L. Boiardi ${ }^{1}$, E. Galli ${ }^{2}$, G. Pazzola $^{1}$, A. Cavazza ${ }^{3}$, G. Restuccia ${ }^{1}$, C. Salvarani ${ }^{4} .{ }^{1}$ Rheumatology Unit, AUSL-IRCCS di Reggio Emilia, Reggio Emilia, Italy; ${ }^{2}$ Rheumatology Unit, University of Modena and Reggio Emilia, Modena, Italy; ${ }^{3}$ Pathology Unit, AUSL-IRCCS di Reggio Emilia, Reggio Emilia, Italy; ${ }^{4}$ Rheumatology Unit, AUSL-IRCCS di Reggio Emilia and University of Modena and Reggio Emilia, Reggio Emilia, Italy

Background: The classification criteria currently used to define giant cell arteritis (GCA) were developed in 1990 by the American College of Rheumatology 